\title{
Polymorphisms in intron 1 of the EGFR gene in non-small cell lung cancer patients
}

\author{
MASAYUKI SHITARA ${ }^{1}$, HIDEFUMI SASAKI ${ }^{1}$, KEISUKE YOKOTA ${ }^{1}$, KATSUHIRO OKUDA ${ }^{1}$, \\ YU HIKOSAKA ${ }^{1}$, SATORU MORIYAMA ${ }^{1}$, MOTOKI YANO ${ }^{1}$, TOMOYA KAWAGUCHI ${ }^{2}$, \\ AKIHITO KUBO $^{2}$, MINORU TAKADA ${ }^{2}$, NAOTO KITAHARA ${ }^{3}$, MEINOSHIN OKUMURA $^{3}$, \\ AKIHIDE MATSUMURA ${ }^{3}, \mathrm{KEIJI} \mathrm{IUCHI}^{3}$ and YOSHITAKA FUJII $^{1}$
}

${ }^{1}$ Department of Oncology, Immunology and Surgery, Nagoya City University Graduate School of Medical Sciences,
Nagoya 467-8601; Departments of ${ }^{2}$ Internal Medicine and ${ }^{3}$ Surgery, National Hospital Organization,
Kinki-chuo Chest Medical Center, Sakai, Osaka 591-8555, Japan

Received October 18, 2011; Accepted December 23, 2011

DOI: 10.3892/etm.2012.681

\begin{abstract}
The epidermal growth factor receptor (EGFR) gene is highly polymorphic and its expression and activity may be affected by various polymorphisms. There have been several studies examining associations between $E G F R$ polymorphisms and clinical outcome of lung cancer therapy; however, the underlying mechanism is largely unknown. The present study investigated EGFR polymorphism status and its correlation with clinicopathological features in Japanese non-small cell lung cancer (NSCLC) patients. We investigated 5 polymorphisms in the EGFR gene $(-216 \mathrm{G} / \mathrm{T},-191 \mathrm{C} / \mathrm{A}$, 8227G/A, D994D and R497K) in 274 surgically-treated NSCLC patients. TaqMan single nucleotide polymorphism (SNP) genotyping assays and a PCR-based assay were used to analyze these polymorphisms. In our cohort of patients we did not find any evidence of the -191C/A polymorphism. Our results showed that the patients with the $8227 \mathrm{GA}$ or AA type in intron 1 had a significantly better prognosis with the antiEGFR therapy than the patients with the GG type $(p=0.0448)$ in terms of recurrence of lung cancer. No significant association was observed between 3 other SNPs (-216G/T, D994D and $\mathrm{R} 497 \mathrm{~K}$ ) and clinicopathological features. The EGFR $8227 \mathrm{G} / \mathrm{A}$ polymorphism in intron 1 may be associated with clinical outcome in NSCLC patients treated with EGFR tyrosine kinase inhibitors.
\end{abstract}

Correspondence to: Dr Hidefumi Sasaki, Department of Oncology, Immunology and Surgery, Nagoya City University Graduate School of Medical Sciences, Kawasumi 1, Mizuho-cho, Mizuho-ku, Nagoya 467-8601, Japan

E-mail: hisasaki@med.nagoya-cu.ac.jp

Key words: epidermal growth factor receptor, polymorphism, lung cancer, gefitinib therapy, $8227 \mathrm{G} / \mathrm{A}$

\section{Introduction}

Lung cancer is a major cause of mortality from malignant diseases due to its high incidence, malignant behavior and lack of major advancements in treatment strategy (1). There is a large body of accumulated evidence that the epidermal growth factor receptor (EGFR) and its family members are heavily involved in the development and progression of numerous human tumors, including lung cancer $(2,3)$. The EGFR tyrosine kinase inhibitor (TKI), gefitinib, was approved for the treatment of non-small cell lung cancer (NSCLC) in Japan in 2002. Two original studies have revealed that EGFR mutation status at the tyrosine kinase (TK) domain in NSCLC patients was correlated with a good response to gefitinib $(4,5)$. From the results of the Iressa Pan-Asia Study (IPASS), EGFR mutations are the strongest predictive biomarker for progression-free survival (PFS) and tumor response to first-line gefitinib therapy for NSCLC (6).

The EGFR gene is highly polymorphic and its expression and activity are significantly affected by various polymorphisms (7-9). As for interethnic differences in CA repeat length in intron 1, a length of less than 17 in Japanese individuals is less frequent than in Caucasians (10). However, the frequency of EGFR mutations is higher in the Japanese population than in other ethnic groups. In intron 1 , the $-216 \mathrm{G} / \mathrm{T}$ and $-191 \mathrm{C} / \mathrm{A}$ polymorphisms in the EGFR promoter are associated with altered promoter activity and gene expression (8). CA simple sequence repeats (CA-SSRs) in intron 1 (rs45559542) $(8,12,13)$, -216G/T (rs712829) $(8,12)$ and D994D (rs2293347) (11) polymorphisms have been reported to influence clinical outcomes in gefitinib-treated NSCLC patients. In addition, the $8227 \mathrm{G} / \mathrm{A}$ polymorphism (rs763317) located in intron 1 has been reported to be associated with smoking status and gender in lung adenocarcinomas in the Taiwanese population (14).

To determine the EGFR polymorphism status and its correlation with clinicopathological features in lung carcinoma in the Japanese population, we investigated EGFR gene status using TaqMan single nucleotide polymorphism (SNP) genotyping assays. These findings were analyzed in relation to the clinicopathologic features of lung cancer. 


\section{Materials and methods}

Patients and treatment. The study group included 261 lung cancer patients who had undergone surgery at the Nagoya City University Hospital, Japan, between 1997 and 2011. Thirty-three patients were treated with gefitinib for the recurrence of lung cancer following surgery. We also investigated polymorphisms for 13 NSCLC patients who had been treated with gefitinib for the recurrence of cancer at the Kinki-chuo Chest Medical Center, Osaka Japan. The lung tumors were classified according to the general rule for clinical and pathological recording of lung cancer in Japan, as well as according to the WHO classification. All tumor samples were immediately frozen and stored at $-80^{\circ} \mathrm{C}$ until assayed.

The clinical and pathological characteristics of the 274 lung cancer patients were as follows: 194 (70.8\%) were male and 80 were female; 192 were diagnosed as adenocarcinoma and 82 were diagnosed as other types of carcinoma (63 squamous cell carcinomas, 6 adenosquamous carcinomas, 6 large cell carcinomas, 3 carcinoids, 3 pleomorphic carcinomas, 1 adenoid cystic carcinoma and 1 carcinosarcoma); 187 (68.2\%) were smokers (current or former smoker) and 87 were non-smokers (Table I). Written informed consent was obtained from the patients and the Institutional Ethics Committee of the Nagoya City University approved the study.

Genotyping assays for the EGFR polymorphism. Genomic DNA was extracted from peripheral blood $(n=109)$ taken prior to surgery or from adjacent normal lung tissues taken at surgery using the Wizard SV Genomic DNA Purification system (Promega Corp., Madison, WI, USA) according to the manufacturer's instructions. EGFR mutation statuses at the kinase domain were investigated using the TaqMan PCR assay (Applied Biosystems, Foster City, CA, USA). The results of the TaqMan PCR assay have been previously reported (15).

TaqMan SNP genotyping assays (Applied Biosystems) were used for genotyping 4 polymorphisms in the EGFR gene (-216G/T, -191C/A, 8227G/A, assay ID: C_2310200_10; and D994D, assay ID: C_15970737_20; Table II) according to the manufacturer's instructions (16). The cycling conditions for the TaqMan SNP assays were as follows: $95^{\circ} \mathrm{C}$ for $10 \mathrm{~min}$, followed by 40 cycles of $95^{\circ} \mathrm{C}$ for $15 \mathrm{sec}$ and $60^{\circ} \mathrm{C}$ for $1 \mathrm{~min}$, with a 1-min extension at $25^{\circ} \mathrm{C}$ following the last cycle. The R521K (rs11543848, also assigned as R497K in the literature) polymorphism was examined by the PCR-RFLP method as described previously (17). Sixty-four lung cancer samples were analyzed for EGFR gene amplification using fluorescence in situ hybridization (FISH) and the results have been previously reported (18).

Statistical analyses. Statistical analyses were carried out using the Mann-Whitney U test for unpaired samples and Wilcoxon's signed rank test for paired samples. Linear relationships between variables were determined by means of simple linear regression. Correlation coefficients were determined by rank correlation using Spearman's test and the $\chi^{2}$ test. The overall survival (OS) of lung cancer patients was examined using the log-rank test. All analyses were performed using the StatView software package (Abacus Concepts Inc, Berkeley, CA, USA) and differences were considered significant when $\mathrm{p}<0.05$.
Table I. Clinical and pathological characteristics of the 274 lung cancer patients.

\begin{tabular}{lrc}
\hline & \multicolumn{2}{c}{ Patients (n=274) } \\
\cline { 2 - 3 } & No. & $\%$ \\
\hline Age (years) & 78 & 28.5 \\
$\leq 60$ & 196 & 71.5 \\
$>60$ & & \\
Gender & 194 & 70.8 \\
Male & 80 & 29.2 \\
Female & & \\
Smoking status & 87 & 31.8 \\
$\quad$ Non-smoker & 187 & 68.2 \\
Smoker & & \\
Pathological subtype & 192 & 70.1 \\
Adeno & 82 & 29.9 \\
$\quad$ Other & & \\
EGFR mutation & 81 & 29.9 \\
Positive & 190 & 70.1 \\
$\quad$ Negative & \\
\hline
\end{tabular}

Smoker, current or former smoker; Adeno, adenocarcinoma; EGFR, epidermal growth factor receptor.

\section{Results}

EGFR gene mutation and amplification statuses. Of the 274 patients, 42 had the deletion-type EGFR mutations in exon 19; 35 had the missense point mutations (5 G719S, 29 L858R and 1 L861Q) in exon 18 or 21; and 4 had exon 20 insertion mutations $(15,20)$. Sixty-four samples were studied for $E G F R$ gene amplification using FISH analyses. According to the criteria by Cappuzzo et al, 21 were FISH-positive and 43 were FISH-negative (19).

EGFR polymorphisms in Japanese lung cancers. In our Japanese cohort, there was no -191C/A polymorphism and we did not perform any further analyses for this polymorphism. For rs712829 (-216G/T), 255 patients were GG, 19 were GT and no TT was found. For rs2293347 (D994D), 125 patients were CC, 110 were CT and 39 were TT. For rs11543848 (R497K), 93 were AA, 135 were GA and 46 were GG. No correlation existed between these 3 SNPs (-216G/T, GG vs. GA+AA; D994D, CC+CT vs. TT; R497K, GG vs. GA+AA) and clinicopathological features of the lung cancers.

Of the 274 patients, 87 had the $8227 \mathrm{G} / \mathrm{A}$ EGFR variant (9 AA and $78 \mathrm{GA})$. Of these, 64 were male and 23 were female, 24 were non-smokers, 59 were smokers and 4 were unknown. Adenocarcinomas were significantly more frequent in GG-type patients $(139 / 187,74.3 \%)$ than in the GA- or AA-type patients $(53 / 87,60.9 \%, p=0.0331)$. However, the polymorphism did not correlate with gender $(\mathrm{p}=0.5687)$, smoking (non-smokers vs. smokers, $\mathrm{p}=0.3325$ ), or $E G F R$ mutation ( $\mathrm{p}=0.1539$ ) statuses of lung cancer (Table III). EGFR gene amplification as identified by FISH positivity was not 
Table II. Genotyping approach for polymorphism analysis of the EGFR gene.

\begin{tabular}{lll}
\hline Primer sequences & $-216 G / T($ rs712829) & -191C/A (rs712830) \\
\hline VIC-MGB & AGCCTCCGCCCCC & CCTCGGCCGCGTCG \\
FAM-MGB & CAGCCTCCTCCCCC & CCTCGGCCGCGGCG \\
Forward primer & CCCGCGCGAGCTAGAC & CCCCGCACGGTGTGA \\
Reverse primer & GGGCGCTCACACCTG & GGCTAGCTCGGGACTCC
\end{tabular}

VIC-MGB, VIC dye-labeled TaqMan MGB probe; FAM-MGB, FAM dye-labeled TaqMan MGB probe; EGFR, epidermal growth factor receptor.

Table III. Association of the EGFR $8227 \mathrm{G} / \mathrm{A}$ polymorphism with clinicopathological data of 274 lung cancer patients.

\begin{tabular}{|c|c|c|c|c|c|}
\hline \multirow[b]{2}{*}{ Factors } & \multicolumn{2}{|c|}{ GG } & \multicolumn{2}{|c|}{$\mathrm{GA}+\mathrm{AA}$} & \multirow[b]{2}{*}{ p-value } \\
\hline & No. & $\%$ & No. & $\%$ & \\
\hline \multicolumn{6}{|l|}{ Age (years) } \\
\hline$\leq 60$ & 52 & 27.8 & 26 & 29.9 & 0.7741 \\
\hline$>60$ & 135 & 72.2 & 61 & 70.1 & \\
\hline \multicolumn{6}{|l|}{ Gender } \\
\hline Male & 130 & 69.5 & 64 & 73.6 & 0.5687 \\
\hline Female & 57 & 30.5 & 23 & 26.4 & \\
\hline \multicolumn{6}{|l|}{ Smoking status } \\
\hline Non-smoker & 63 & 33.7 & 24 & 27.6 & 0.3325 \\
\hline Smoker & 124 & 66.3 & 59 & 72.4 & \\
\hline \multicolumn{6}{|c|}{ Pathological subtype } \\
\hline Adeno & 139 & 74.3 & 53 & 60.9 & 0.0331 \\
\hline Others & 48 & 25.7 & 34 & 39.1 & \\
\hline \multicolumn{6}{|l|}{ EGFR mutation } \\
\hline Positive & 61 & 32.6 & 20 & 23.8 & 0.1539 \\
\hline Negative & 126 & 67.4 & 64 & 76.2 & \\
\hline
\end{tabular}

Smoker, current smoker or former smoker; Adeno, adenocarcinoma; $E G F R$, epidermal growth factor receptor.

correlated with polymorphism statuses, including D994D $(\mathrm{p}=0.5884),-216 \mathrm{G} / \mathrm{T}(\mathrm{p}>0.9999), \mathrm{R} 497 \mathrm{~K}(\mathrm{p}=0.2043)$ and $8227 \mathrm{G} / \mathrm{A}(\mathrm{p}>0.9999)$.

Correlation between clinical course of lung cancer patients and EGFR polymorphisms. The OS of the 225 lung cancer patients who did not receive gefitinib, with follow-up until June 30, 2011, was studied in reference to the EGFR polymorphism status. The prognosis was not significantly different between the $E G F R$ 8227G/A types (GA+AA, 23/73 were deceased; GG, 53/152 were deceased; $p=0.1753$; Fig. 1). No significant association was observed between the other 3 SNPs $(-216 \mathrm{G} / \mathrm{T}$, D994D and $\mathrm{R} 497 \mathrm{~K}$ ) and disease outcome (data not shown).

Correlation between clinical course of gefitinib-treated lung cancer patients and EGFR polymorphism. The OS of 46 gefitinib-treated lung cancer patients, with follow-up until June 30, 2011, was studied in reference to the EGFR polymorphism status. In this analysis, 12 patients had EGFR 8227GA

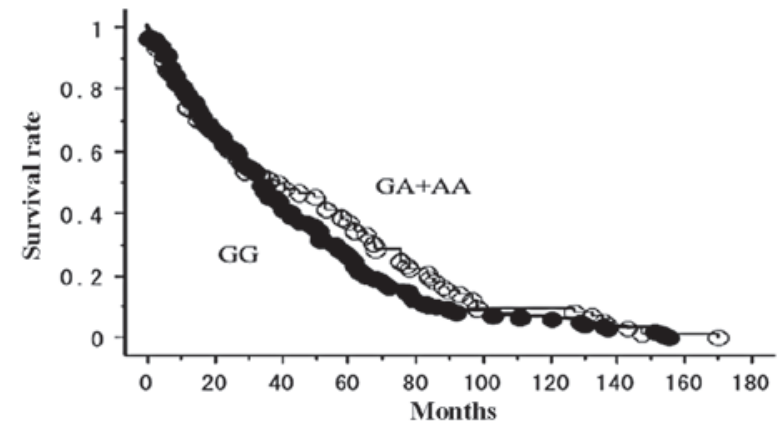

Figure 1. The overall survival of 225 lung cancer patients who were not treated with gefitinib was studied in reference to the $E G F R$ polymorphism $(8227 \mathrm{G} / \mathrm{A})$ status. The prognosis was not significantly different between the patients with $8227 \mathrm{GG}$ type (53/152 were deceased) and the patients with $8227 \mathrm{GA}$ or AA type (23/73 were deceased) (log-rank test, $\mathrm{p}=0.1753) . E G F R$, epidermal growth factor receptor.

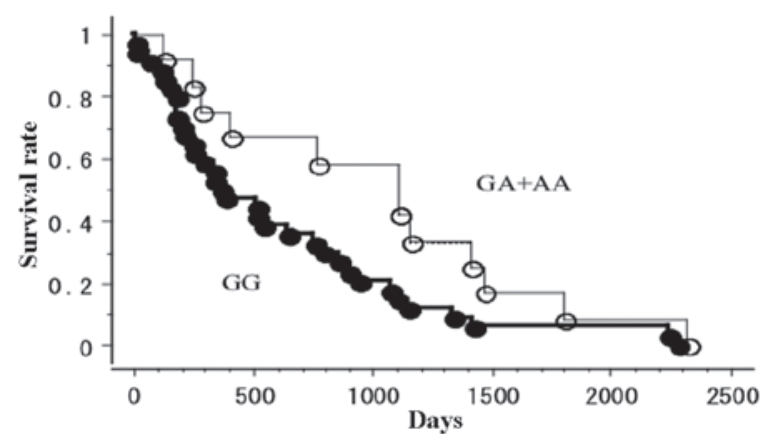

Figure 2. The overall survival of 46 gefitinib-treated lung cancer patients was studied in reference to the EGFR polymorphism (8227G/A) status. The patients with $8227 \mathrm{GA}$ or AA type (5/12 were deceased; median follow-up, 33.3 months) had significantly better prognosis than the patients with $8227 \mathrm{GG}$ type $(26 / 34$ were deceased; median follow-up, 20.0 months) (log-rank test, $\mathrm{p}=0.0448$ ) $E G F R$, epidermal growth factor receptor.

or AA types. Of the 46 patients, 31 had EGFR mutations and 11 were EGFR $8227 \mathrm{GA}$ or AA. There was a tendency towards higher $E G F R$ mutation ratio in the $8227 \mathrm{GA}$ - or AA-type patients compared with GG-type patients $(\mathrm{p}=0.0702)$. Other clinical backgrounds, including gender $(\mathrm{p}=0.3071)$, smoking $(\mathrm{p}=0.4893)$ and pathological status $(\mathrm{p}=0.3059)$ were not correlated with $8227 \mathrm{G} / \mathrm{A}$ polymorphism status. The prognosis following gefitinib therapy was significantly better for the $E G F R$ GA- or AA-type patients (5/12 were deceased; mean survival, 1,014 days) when compared with the $8227 \mathrm{GG}$-type patients (26/34 were deceased; mean survival, 607 days; log-rank test, 
$\mathrm{p}=0.0448$; Fig. 2). Using the multivariate analysis, EGFR mutation $(\mathrm{p}=0.0316$; hazard ratio, 2.174) but not $8227 \mathrm{G} / \mathrm{A}(\mathrm{p}=0.2232$; hazard ratio, 1.587) was the independent prognostic factor for gefitinib-treated patients.

There was no association between the other 3 polymorphisms $(-216 \mathrm{G} / \mathrm{T}, \mathrm{p}=0.7599$; D994D, $\mathrm{p}=0.1813$; and R497K, $\mathrm{p}=0.885)$ and prognosis for the gefitinib-treated patients.

\section{Discussion}

In this study, gefitinib-treated patients with an A allele at the EGFR $8227 \mathrm{G} / \mathrm{A}$ site were found to have a better prognosis compared with GG-type patients. However, there was no association between the other 3 polymorphisms (-216G/T, D994D and $\mathrm{R} 497 \mathrm{~K}$ ) and prognosis following gefitinib therapy.

Previous studies have suggested that $-216 \mathrm{G} / \mathrm{T}(8,12)$ and D994D (11) polymorphisms are associated with clinical outcome of gefitinib therapy. In intron 1, CA-SSR of EGFR has been the most studied polymorphism. CA-SSR has been associated with EGFR gene expression and has been reported to correlate with clinical outcome of gefitinib therapy $(8,12,13,21)$. Shorter CA repeats have been associated with higher transcription levels of EGFR and have been reported to be correlated with better clinical outcome of gefitinib therapy. Tiseo et al revealed that patients with the CA-16 genotype had a longer survival compared with those with other genotypes (13). Liu et al found that the $-216 \mathrm{G} / \mathrm{T}$ polymorphism and CA-19 genotype are found more frequently in patients with exon 19 deletions (22). On the other hand, Suzuki et al reported that the EGFR protein expression level was significantly higher in the shorter CA repeats group than in the longer allele group, but its length was not associated with EGFR somatic mutations (23). In a Japanese cohort, Ichihara et al reported that patients with a short CA-SSR1 had a prolonged OS as compared with those with a longer CA-SSR, but this difference was not significant in patients with a drug-sensitive EGFR mutation (p=0.13) (24). They found that FISH status, CA-SSR1 length and the SNP status in the promoter region $(-216 \mathrm{G} / \mathrm{T}$ or $-191 \mathrm{G} / \mathrm{A})$ had no association with responsiveness to gefitinib in cases of lung cancer in Japanese individuals, similar to our results. One explanation for the results is that the variant forms of the SNPs, -216 G/T (6.6\%) and -191G/A (0.6\%), were less frequent in East Asians than in individuals of European descent $(60.3$ and 37\%, respectively) (25). As for the D994D polymorphism, using direct sequencing, our group has previously revealed that the polymorphism did not affect the gefitinib sensitivity in Japanese individuals (26). This polymorphism is located in exon 25 and a synonymous SNP does not change the amino acid sequence of the protein, so it does not influence the biological function of the protein itself. Ma et al revealed that the D994D polymorphism did affect PFS but not OS following gefitinib therapy (11).

The $8227 \mathrm{G} / \mathrm{A}$ polymorphism is also located in intron 1 , but there have been few studies examining this $\operatorname{SNP}(14,27)$. Jou et al revealed that the EGFR $8227 \mathrm{G} / \mathrm{A}$ polymorphism was associated with lung cancer, especially in non-smoking female lung adenocarcinoma patients in the Taiwanese population (14). Thus, this variation may lead to the different modifications of cancer genes, including EGFR, in tumorigenic pathways among different histological subtypes, gender and ethnicity. The $8227 \mathrm{G} / \mathrm{A}$ SNP is located in intron $1,6.9 \mathrm{~kb}$ downstream of the CA-SSR1 polymorphism. Additional functional analyses of this SNP are needed to better understand the mechanism by which the $8227 \mathrm{G} / \mathrm{A}$ SNP of EGFR affects lung cancer. In our analysis, although the $8227 \mathrm{G} / \mathrm{A}$ polymorphism in intron 1 was not correlated with EGFR somatic mutations, the GA or AA type was associated with longer survival of the gefitinib-treated patients. The underlying mechanisms remain unclear, but it may be that intron 1 of EGFR is associated with sensitivity to EGFR TKIs in lung cancer patients, and is correlated with certain biomarkers other than EGFR mutations. The sample size of the present study was too small to address this hypothesis. The extact effect of the polymorphism on survival time of patients treated with or without EGFR TKIs needs further clinical investigation with a larger sample size.

In summary, the $8227 \mathrm{G} / \mathrm{A}$ polymorphism of EGFR may influence OS in gefitinib-treated lung cancer patients.

\section{Acknowledgements}

The authors thank Mrs. Miki Mochizuki for her excellent technical assistance. This study was supported by Grants-in-Aid for Scientific Research, Japan Society for the Promotion of Science (JSPS) (Nos. 23659674, 21390394 and 21591820) and a grant for cancer research from the Program for developing the supporting system for upgrading education and research (2009) from the Ministry of Education, Culture, Sports, Science and Technology of Japan.

\section{References}

1. Ginsberg RJ, Kris K and Armstrong G: Cancer of the lung. In: Principles and Practice of Oncology. DeVita VT, Hellman S and Rosenberg SA (eds). 4th edition. JB Lippincott Co., Philadelphia, PA, pp673-682, 1993.

2. Nicholson RI, Gee JM and Harper ME: EGFR and cancer prognosis. Eur J Cancer 37: S9-S15, 2001.

3. Onn A, Choe DH, Herbst RS, et al: Synchronous overexpression of epidermal growth factor receptor and HER2-neu protein is a predictor of poor outcome in patients with stage I non-small cell lung cancer. Clin Cancer Res 10: 136-143, 2004.

4. Paez JG, Jänne PA, Lee JC, et al: EGFR mutations in lung cancer: correlation with clinical response to gefitinib therapy. Science 304: 1497-1500, 2004.

5. Lynch TJ, Bell DW, Sordella R, et al: Activating mutations in the epidermal growth factor receptor underlying responsiveness of non-small-cell lung cancer to gefitinib. N Engl J Med 350: 2129-2139, 2004.

6. Fukuoka M, Wu Y-L, Thongprasert S, et al: Biomarker analyses and first overall survival results from a phase III, randomized, open-label, first-line study of gefitinib versus carboplatin/paclitaxel in clinically selected patients with advanced non-small-cell lung cancer in Asia (IPASS). J Clin Oncol 29: 2866-2874, 2011.

7. Liu W, Innocenti F, Wu MH, et al: A functional common polymorphism in a Sp1 recognition site of the epidermal growth factor receptor gene promoter. Cancer Res 65: 46-53, 2005.

8. Liu W, Wu X, Zhang W, et al: Relationship of EGFR mutations, expression, amplification, and polymorphisms to epidermal growth factor receptor inhibitors in the NCI60 cell lines. Clin Cancer Res 13: 6788-6795, 2007.

9. Brandt B, Meyer-Staeckling S, Schmidt H, et al: Mechanisms of EGFR gene transcription modulation: relationship to cancer risk and therapy response. Clin Cancer Res 12: 7252-7260, 2006.

10. Liu W, Innocenti F, Chen P, et al: Interethnic difference in the allelic distribution of human epidermal growth factor receptor intron 1 polymorphism. Clin Cancer Res 9: 1009-1012, 2003.

11. Ma F, Sun T, Shi Y, et al: Polymorphisms of EGFR predict clinical outcome in advanced non-small-cell lung cancer patients treated with Gefitinib. Lung Cancer 66: 114-119, 2009. 
12. Liu G, Gurubhagavatula S, Zhou W, et al: Epidermal growth factor receptor polymorphisms and clinical outcomes in non-small-cell lung cancer patients treated with gefitinib. Pharmacogenomics J 8: 129-138, 2008.

13. Tiseo M, Capelletti M, De Palma G, et al: Epidermal growth factor receptor intron-1 polymorphism predicts gefitinib outcome in advanced non-small cell lung cancer. J Thorac Oncol 3: 1104-1111, 2008.

14. Jou YS, Lo YL, Hsiao CF, et al: Association of an EGFR intron 1 SNP with never smoking female lung adenocarcinoma patients. Lung Cancer 64: 251-256, 2009.

15. Endo K, Konishi A, Sasaki H, et al: Epidermal growth factor receptor gene mutation in non-small cell lung cancer using highly sensitive and fast TaqMan PCR assay. Lung Cancer 50: 375-384, 2005

16. De la Vega FM, Lazaruk KD, Rhodes MD and Wenz MH: Assessment of two flexible and compatible SNP genotyping platforms: TaqMan SNP Genotyping Assays and the SNPlex Genotyping System. Mutat Res 573: 111-135, 2005.

17. Sasaki H, Okuda K, Shimizu S, et al: EGFR R497K polymorphism is a favorable prognostic factor for advanced lung cancer. J Cancer Res Clin Oncol 135: 313-318, 2009.

18. Sasaki H, Shimizu S, Okuda K, et al: Epidermal growth factor receptor gene amplification in surgical resected Japanese lung cancer. Lung Cancer 64: 295-300, 2009.

19. Cappuzzo F, Hirsch FR, Rossi E, et al: Epidermal growth factor receptor gene and protein and gefitinib sensitivity in non-smallcell lung cancer. J Natl Cancer Inst 97: 643-655, 2005.

20. Sasaki H, Endo K, Takada M, et al: EGFR exon 20 insertion mutation in Japanese lung cancer. Lung Cancer 58: 324-328, 2007.
21. Nie Q, Wang Z, Zhang GC, et al: The epidermal growth factor receptor intron 1 (CA) $n$ microsatellite polymorphism is a potential predictor of treatment outcome in patients with advanced lung cancer treated with Gefitinib. Eur J Pharmacol 570: 175-181, 2007.

22. Liu W, He L, Ramírez J, et al: Functional EGFR germline polymorphisms may confer risk for EGFR somatic mutations in non-small cell lung cancer, with a predominant effect on exon 19 microdeletions. Cancer Res 71: 2423-2427, 2011.

23. Suzuki M, Kageyama S, Shinmura K, et al: Inverse relationship between the length of the EGFR CA repeat polymorphism in lung carcinoma and protein expression of EGFR in the carcinoma. J Surg Oncol 98: 457-461, 2008.

24. Ichihara S, Toyooka S, Fujiwara Y, et al: The impact of epidermal growth factor receptor gene status on gefitinib-treated Japanese patients with non-small-cell lung cancer. Int J Cancer 120: 1239-1247, 2007.

25. Nomura M, Shigematsu H, Li L, et al: Polymorphisms, mutations, and amplification of the EGFR gene in non-small cell lung cancers. PLos One 4: 715-727, 2007.

26. Sasaki H, Okuda K, Takada M, et al: A novel EGFR mutation $\mathrm{D} 1012 \mathrm{H}$ and polymorphism at exon 25 in Japanese lung cancer. J Cancer Res Clin Oncol 134: 1371-1376, 2008.

27. Girard N, Lou E, Azzoli CG, et al: Analysis of genetic variants in never-smokers with lung cancer facilitated by an internet-based blood collection protocol: a preliminary report. Clin Cancer Res 16: 755-763, 2010 